University of New Hampshire

University of New Hampshire Scholars' Repository

Physics Scholarship

Physics

$9-1-2008$

\title{
Posteruptive phenomena in coronal mass ejections and substorms: Indicators of a universal process?
}

K. K. Reeves

T. B. Guild

W. J. Hughes

K. E. Korreck

J. Lin

See next page for additional authors

Follow this and additional works at: https://scholars.unh.edu/physics_facpub

Part of the Physics Commons

\section{Recommended Citation}

Reeves, K. K., et al. (2008), Posteruptive phenomena in coronal mass ejections and substorms: Indicators of a universal process? J. Geophys. Res., 113, A00B02, doi:10.1029/2008JA013049.

This Article is brought to you for free and open access by the Physics at University of New Hampshire Scholars' Repository. It has been accepted for inclusion in Physics Scholarship by an authorized administrator of University of New Hampshire Scholars' Repository. For more information, please contact Scholarly.Communication@unh.edu. 


\section{Authors}

K. K. Reeves, T. B. Guild, W. J. Hughes, K. E. Korreck, J. Lin, J. Raymond, S. Savage, Nathan A. Schwadron, Harlan E. Spence, D. F. Webb, and M. Wiltberger 


\title{
Posteruptive phenomena in coronal mass ejections and substorms: Indicators of a universal process?
}

\author{
K. K. Reeves, ${ }^{1}$ T. B. Guild, ${ }^{2}$ W. J. Hughes,${ }^{3}$ K. E. Korreck, ${ }^{1}$ J. Lin, ${ }^{1}$ J. Raymond, ${ }^{1}$ \\ S. Savage, ${ }^{4}$ N. A. Schwadron, ${ }^{3}$ H. E. Spence, ${ }^{3}$ D. F. Webb, ${ }^{5}$ and M. Wiltberger ${ }^{6}$ \\ Received 23 January 2008; revised 1 April 2008; accepted 16 April 2008; published 9 July 2008.
}

[1] We examine phenomena associated with eruptions in the two different regimes of the solar corona and the terrestrial magnetosphere. We find striking similarities between the speeds of shrinking magnetic field lines in the corona and dipolarization fronts traversing the magnetosphere. We also examine the similarities between supra-arcade downflows observed during solar flares and bursty bulk flows seen in the magnetotail and find that these phenomena have remarkably similar speeds, velocity profiles, and size scales. Thus we show manifest similarities in the magnetic reconfiguration in response to the ejection of coronal mass ejections in the corona and the ejection of plasmoids in the magnetotail. The subsequent return of loops to a quasi-potential state in the corona and field dipolarization in the magnetotail are physical analogs and trigger similar phenomena such as downflows, which provides key insights into the underlying drivers of the plasma dynamics.

Citation: Reeves, K. K., et al. (2008), Posteruptive phenomena in coronal mass ejections and substorms: Indicators of a universal process?, J. Geophys. Res., 113, A00B02, doi:10.1029/2008JA013049.

\section{Introduction}

[2] Magnetic reconnection has been defined as the topological reordering of magnetic field lines caused by a local disruption of the ideal conditions that keep the plasma frozen to the magnetic field [Biskamp, 2000; Priest and Forbes, 2000]. Magnetic reconnection allows rapid conversion of magnetic energy into kinetic and thermal energy, and thus it plays an important role in astrophysical plasmas throughout the universe, including in our local environment. The Earth's magnetosphere and the corona of the Sun are two local environments where magnetic reconnection is an important process. Magnetic reconnection is the favored explanation for solar flares because it is the only mechanism that satisfactorily explains observational phenomena that occur during flares, including separating flare ribbons seen in $\mathrm{H} \alpha$, cusp-shaped arches of soft X-ray loops, and hard Xray sources at flare loop tops and footpoints [e.g., Priest and Forbes, 2002]. Similarly, magnetic reconnection is also a viable explanation for substorms, since it is consistent with the sudden release of energy, the dynamics of the growth

\footnotetext{
${ }^{1}$ Harvard-Smithsonian Center for Astrophysics, Cambridge, Massachusetts, USA.

${ }^{2}$ The Aerospace Corporation, Chantilly, Virginia, USA. USA.

${ }^{3}$ Department of Astronomy, Boston University, Boston, Massachusetts,

${ }^{4}$ Department of Physics, Montana State University, Bozeman, Montana, USA.

${ }^{5}$ Institute for Scientific Research, Boston College, Chestnut Hill, Massachusetts, USA.

${ }^{6}$ High Altitude Observatory, National Center for Atmospheric Research, Boulder, Colorado, USA.
}

Copyright 2008 by the American Geophysical Union. 0148-0227/08/2008JA013049 phase and expansion phase onset, and the topological magnetic field changes that have been observed to accompany substorms [e.g., Coroniti, 1985].

[3] In this paper, we draw parallels between posteruption phenomena that take place in long duration solar flares and substorms [see, e.g., Priest and Forbes, 2000]. The coronal phenomena we focus on are predominantly found in longduration flares (the class of flares commonly associated with coronal mass ejections) rather than compact or confined flares, although magnetic reconnection is probably involved in both flare classes. Figure 1 shows schematic drawings of magnetic reconnection events in the magnetosphere and corona that lead to the posteruption phenomena that we will study. Analogies between solar flares and magnetospheric substorms have been drawn before [e.g., Akasofu, $1979,1985]$, but in this work we focus on recent, more detailed observations of both phenomena.

[4] One hurdle that must be overcome before we can make fruitful comparisons of solar and magnetospheric phenomena is one of nomenclature. The magnetospheric and solar communities use different terms for phenomena that are actually the same when the underlying physics is considered. Often these terms are based on prominent morphological or phenomenological characteristics present in the original observations. For instance, magnetic reconnection is widely accepted in the solar community as the driver of solar flares, and a consequence of this reconnection is the relaxation of magnetic field lines into a more potential shape. This process was originally termed "field line shrinkage" by Švestka et al. [1987] because of the way that field lines shrink in height as they become more potential. The magnetospheric community, on the other hand, refers to the relaxation of a stretched magnetic field 

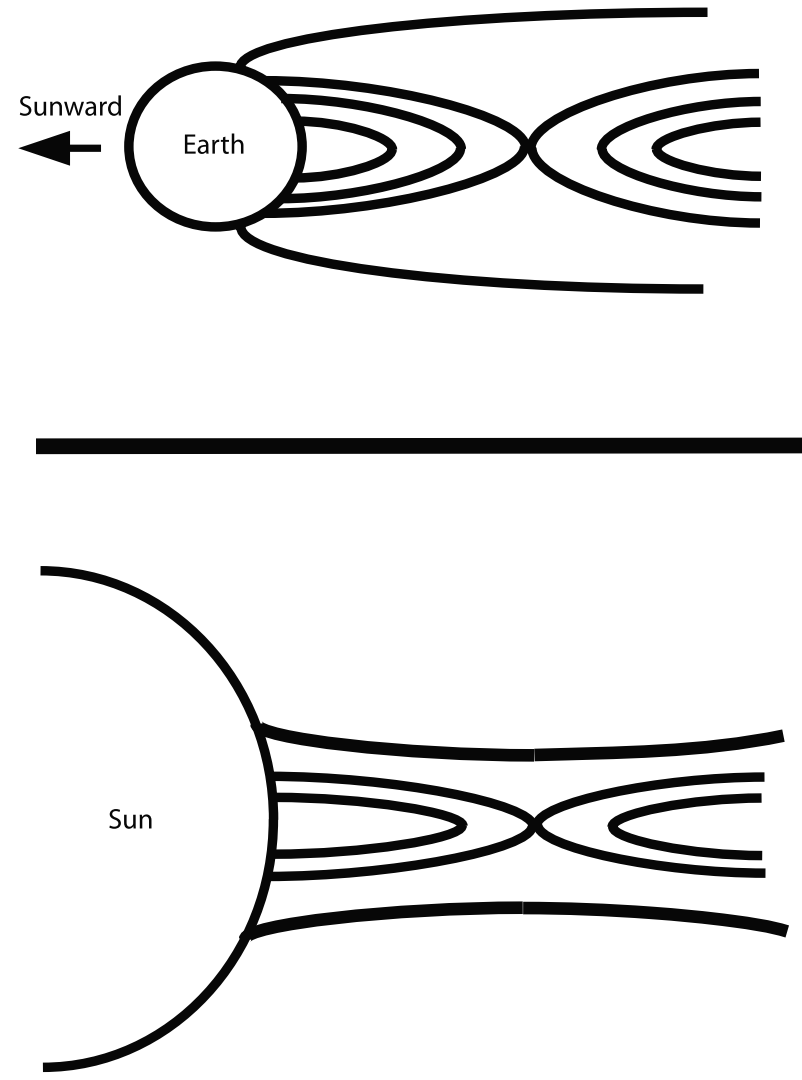

Figure 1. (top) The magnetosphere with a magnetotail reconnection site and (bottom) a solar flare reconnection point in the corona. The drawings are not to scale although the systems do have similar length scales.

into a field that is more dipolar (i.e., more potential) as dipolarization. In essence, dipolarization and field line shrinkage are terms that refer to the same phenomena.

[5] Another obvious challenge when comparing solar and magnetospheric phenomena is that the kinds of observations that are possible in each environment lead to measurements of very different parameters. In the magnetosphere, many satellites (e.g., IMP satellites, Cluster, GEOTAIL, THEMIS) can measure in situ flows and magnetic fields. These measurements give a wealth of information about the state of the local plasma but give little or no information about the spatial variations of the plasma parameters or global morphologies. Observations of the corona are done remotely via imaging or spectroscopic instruments in space or on the ground. These instruments can give temperature and density information about the plasma on the Sun, although generally at spatial resolutions of no better than one $\operatorname{arsec}(725 \mathrm{~km})$. These instruments also provide good spatial information about the overall morphological features of solar phenomena. However, information about the magnetic field in the corona is lacking in these measurements, and thus the magnetic field can only be inferred though motions of the plasma or extrapolations from measurements of the magnetic field in the photosphere. Furthermore, the distance along the line of sight is often difficult to infer, so in most cases the spatial information is reduced to two dimensions.

[6] We cannot blindly compare all the phenomena that we see. We must look for defining physical parameters that will allow us to accurately represent the system. In both substorms and flares, magnetic reconnection plays a key role, thus we will focus on quantities associated with reconnection processes. The Alfvén speed, length scales, plasma $\beta$, magnitude of the magnetic field, and the density of the surrounding plasma are all considered in the following discussions. In Table 1, we give typical plasma parameters for the magnetosphere and the corona. Although the plasma density, magnetic field strength, and plasma beta are all orders of magnitude different in the corona and magnetosphere, the Alfvén speeds (and hence the Alfvén Mach number) and the length scales are remarkably similar. We shall argue that it is these quantities that govern the behavior of similar posteruption phenomena observed in both the corona and the magnetosphere.

[7] In section 2 we compare observational results pertaining to field line shrinkage in the corona and dipolarization in the magnetosphere. In section 3, we compare supra-arcade downflows seen above postflare arcades with bursty bulk flows in the magnetosphere. Discussion and conclusions are presented in section 4 .

\section{Field Line Shrinkage and Dipolarization}

[8] The term "shrinkage" for flares in the solar corona was coined by Švestka et al. [1987], who noticed that cool postflare loops observed in $\mathrm{H}-\alpha$ never reached the heights of hotter loops observed in X rays. They postulated that the field lines containing the radiating plasma must have shrunk as they cooled, so that we observe the cool loops at lower altitudes than the hot ones. There have been several subsequent observations of field line shrinkage in solar flares and coronal arches using SXT on Yohkoh [Forbes and Acton, 1996; Hiei and Hundhausen, 1996], RHESSI [Sui and Holman, 2003; Sui et al., 2004; Veronig et al., 2006] and the X-Ray Telescope (XRT) on the Hinode mission [Reeves et al., 2008]. These studies show that the speed of the field line shrinkage is on the order of $50 \mathrm{~km} \mathrm{~s}^{-1}$ for loops with high altitudes above the solar surface and can be as low as $2-3 \mathrm{~km} \mathrm{~s}^{-1}$ for lower-lying loops.

[9] An example of an XRT observation of field line shrinkage for a flare that occurred on 5 May 2007 is shown in Figure 2. This figure shows two images of a set of flaring loops on the disk, taken about 9 min apart. The bright loops in the arcade in the earlier image, taken at 2236:32 UT, are cusp-shaped. In the later image, taken at 2245:50 UT, the loops have a more rounded shape. A difference image of the two data sets (also shown in Figure 2) clearly shows the difference in loop shape and altitude. This shape change is indicative of the field lines relaxing (shrinking) to a more potential state. The speed of the field line shrinkage was found to be about $5 \mathrm{~km} \mathrm{~s}^{-1}$ for the loops shown in Figure 2,

Table 1. Plasma Parameters for the Magnetosphere and the Corona

\begin{tabular}{lcc}
\hline & Corona & Magnetosphere \\
\hline Temperature & $10^{6} \mathrm{~K}$ & $10^{7} \mathrm{~K}$ \\
Density & $10^{10} \mathrm{~cm}^{-3}$ & $1 \mathrm{~cm}^{-3}$ \\
Magnetic field & $10-100 \mathrm{G}$ & $10^{-3} \mathrm{G}$ \\
Plasma $\beta$ & $0.001-0.01$ & 1 \\
Length scale & $1000 \mathrm{~km}$ & $1000 \mathrm{~km}$ \\
Alfvén speed & $100-1000 \mathrm{~km} \mathrm{~s}^{-1}$ & $100-1000 \mathrm{~km} \mathrm{~s}^{-1}$ \\
\hline
\end{tabular}



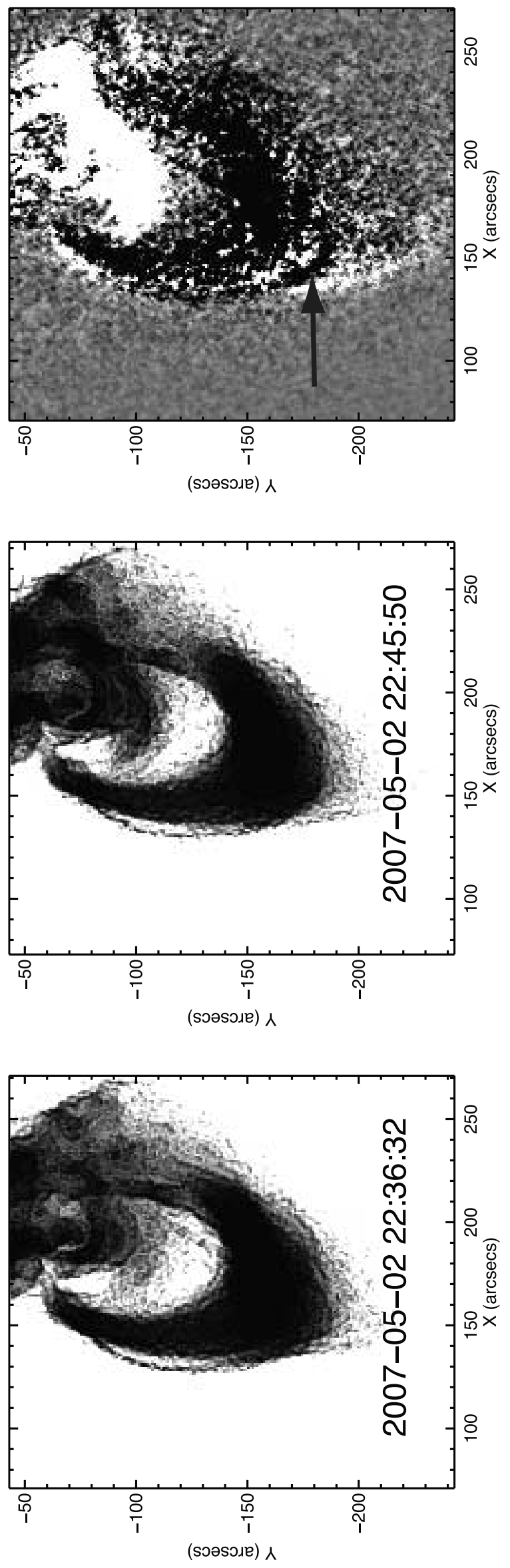

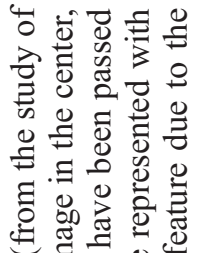

.

so 0

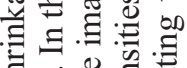

के ठ⿺辶

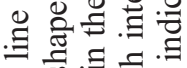

可

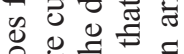

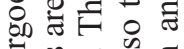

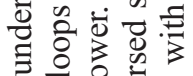

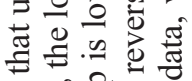

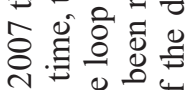

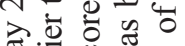

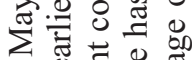

츄 호을

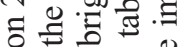

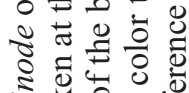

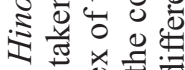

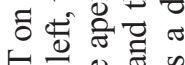

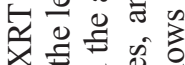

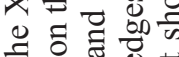

I 8 ती 0

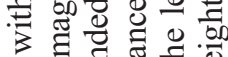

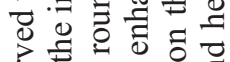

记 0 。

०ें

잉

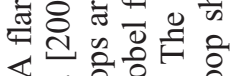

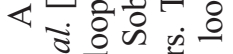

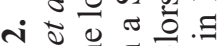

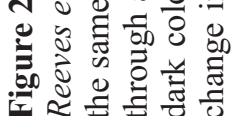




\section{MARCH I}

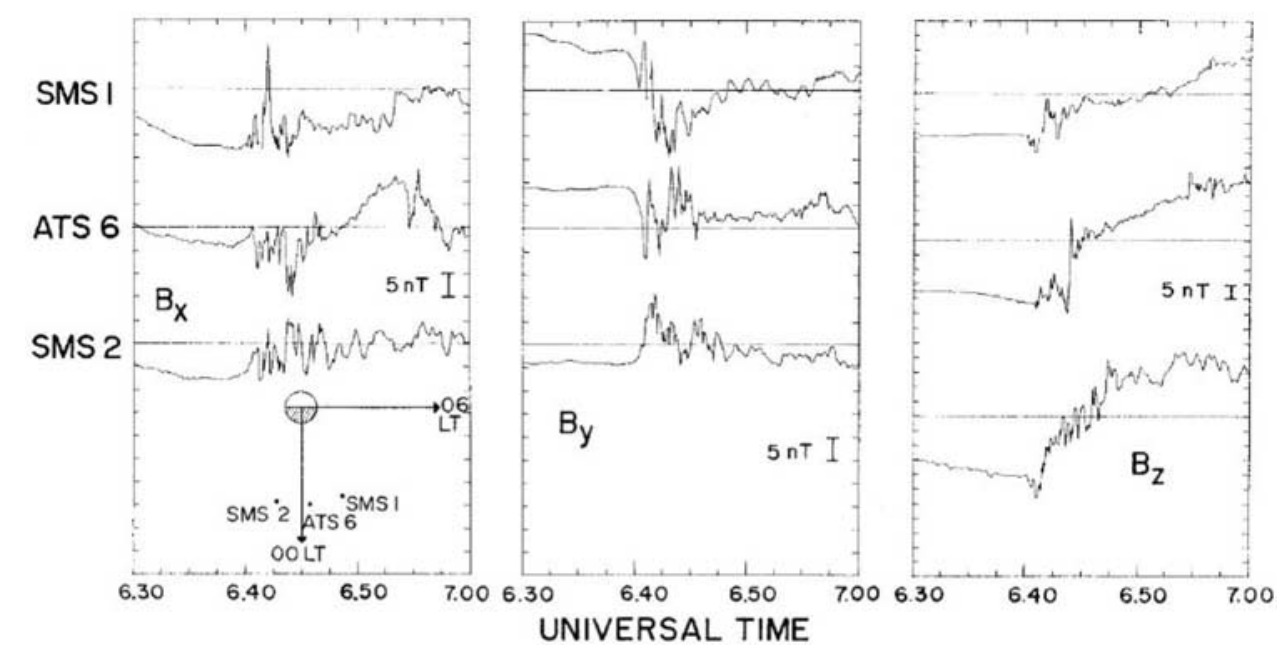

Figure 3. An example of an observation of dipolarizing field lines in the magnetosphere, taken from the study of Hughes [1988]. The figure shows magnetometer data from three spacecraft, spaced about 20 degrees apart. Dipolarization is indicated by an increase in the $\mathrm{B}_{z}$ component of the field.

and fainter, higher altitude loops were found to shrink at a speed of about $50 \mathrm{~km} \mathrm{~s}^{-1}$ in this same event [Reeves et al., 2008].

[10] Observations of dipolarization in the magnetosphere are necessarily somewhat indirect, since measurements are made in situ by spacecraft orbiting the Earth, allowing detailed observations of the plasmas and fields locally but making the global context difficult to discern. Nonetheless, dipolarization has been inferred from observations of increases in the pole-aligned magnetic field [e.g., Hughes and Singer, 1985; Hughes, 1988; Liou et al., 2002]. This dipolarization is accompanied by a disruption of the crosstail current [McPherron et al., 1973]. An example of a dipolarization observation in the magnetosphere from the study of Hughes [1988] is shown in Figure 3. Figure 3 shows magnetometer data from three satellites in geosychronous orbit during a substorm onset. Increases in the $\mathrm{B}_{z}$ component of the field indicate that the field is becoming more dipolar, since $B_{z}$ points in the direction of the Earth's dipole.

[11] Speeds of the dipolarization front are difficult to observe in the magnetosphere because of the in situ nature of the measurements, but multispacecraft data have recorded dipolarization speeds of $100-200 \mathrm{~km} \mathrm{~s}^{-1}$ at $8-9 \mathrm{R}_{E}$ [Ohtani, 1998], and recent analyses of multispacecraft data from the Cluster mission have determined the dipolarization speed in one event to be about $25 \mathrm{~km} \mathrm{~s}^{-1}$ at $5 \mathrm{R}_{E}$ [Apatenkov et al., 2007] and $77 \mathrm{~km} \mathrm{~s}^{-1}$ at $14.5 \mathrm{R}_{E}$ in another event [R. Nakamura et al., 2002]. Thus there is considerable overlap between the speeds observed for dipolarization fronts in the magnetosphere, and the speeds of shrinking magnetic fields observed during a flare in the corona.

[12] Field line shrinkage, or dipolarization, following solar flares and substorms is an expected result of reconnection [Forbes, 1996; Coroniti, 1985]. Using a two-dimensional model of reconnection in solar flares, Lin [2004] found that loops shrink dramatically in the early phase of their evolution, just after the reconnection occurs, and that loops reconnected shortly after flare initiation will shrink more than loops reconnected later in the event. The trajectories and velocities of the loop tops in this model are shown in Figure 4. Even though this model was originally designed for flares in the corona, it does a reasonable job of qualitatively describing dipolarization in the magnetosphere, and it is able to quantitatively predict dipolarization speeds as well; the loop top speeds start at $100-200 \mathrm{~km} \mathrm{~s}^{-1}$ for loops that are formed at $4-6 \times 10^{4} \mathrm{~km}$ (i.e., 6-10 $\mathrm{R}_{E}$ ).

[13] Although the dipolarization process is similar in the magnetosphere and the corona, differences in the geometries of the two systems have effects that should be mentioned. For example, in the magnetosphere, the magnetic field configuration can become far more stretched before the initiation of a substorm than the coronal loops formed in the course of a solar eruption; magnetospheric field lines typically lose up to $80 \%$ of their initial height during the dipolarization process, whereas loops in the corona typically lose more like $20 \%$ of their initial height [Forbes and Acton, 1996; Reeves et al., 2008]. Additionally, dipolarizing magnetic field lines in the magnetosphere eventually return to the dayside (whereas flare loops in the corona have no such escape option), although this process may not have a significant effect on the dynamics of the dipolarization of the loops in the magnetosphere.

[14] Another similarity between the two systems manifests at the footpoints of the dipolarizing loops. The geotail field lines are anchored in the relatively cool, dense ionosphere in each hemisphere, as shown in Figure 1. Some of the energy released in the reconnection process is converted into accelerated electrons that generate the visible aurora at both ends of the field line. The two "auroral ribbons" are made visible by the nonthermal optical and UV auroral emissions. The fraction of the energy released via reconnection that is deposited in the ionosphere by electron precipitation and joule heating generates beams of ionospheric ions that flow up into the magnetosphere along auroral field lines. These ions are accelerated via a multi- 


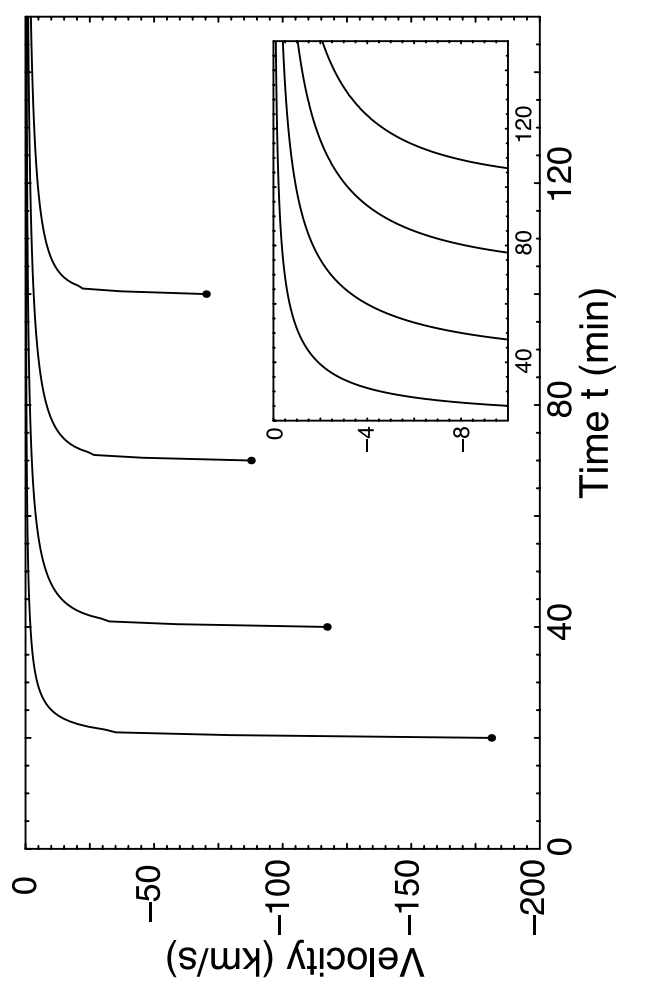

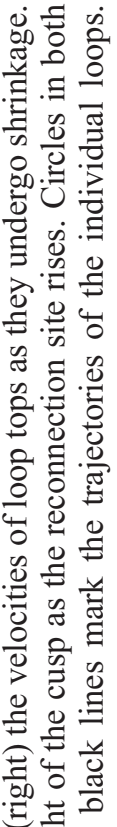

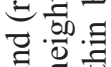

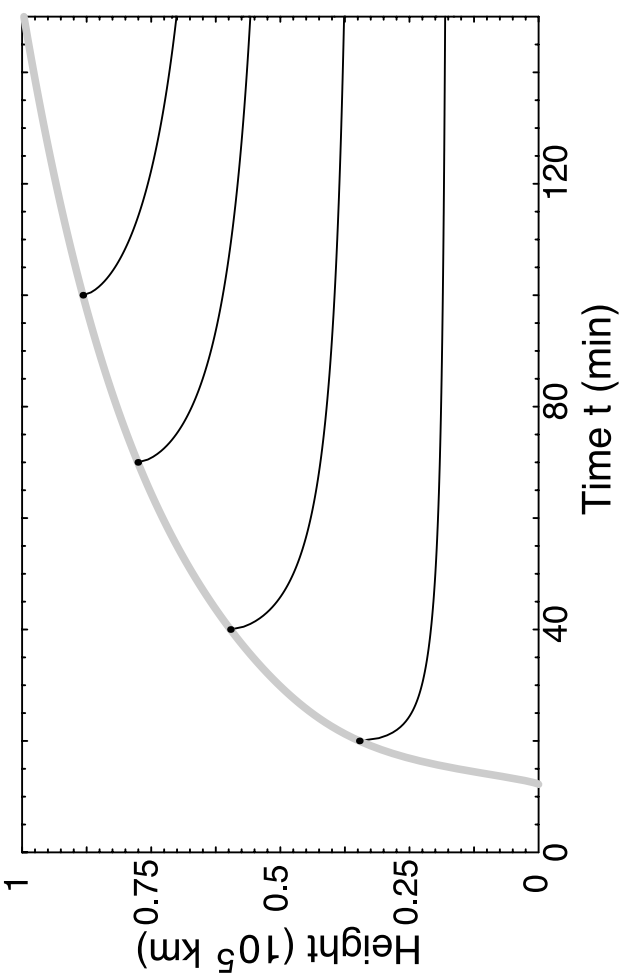

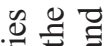

o

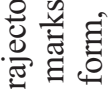

娄

등

范

ᄃ

을

.

흘 흥. 을

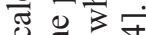

त

.$\Xi$ \&

。․․․․․

ㄹㅣㄹㅀ

का

广்

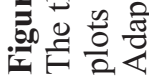




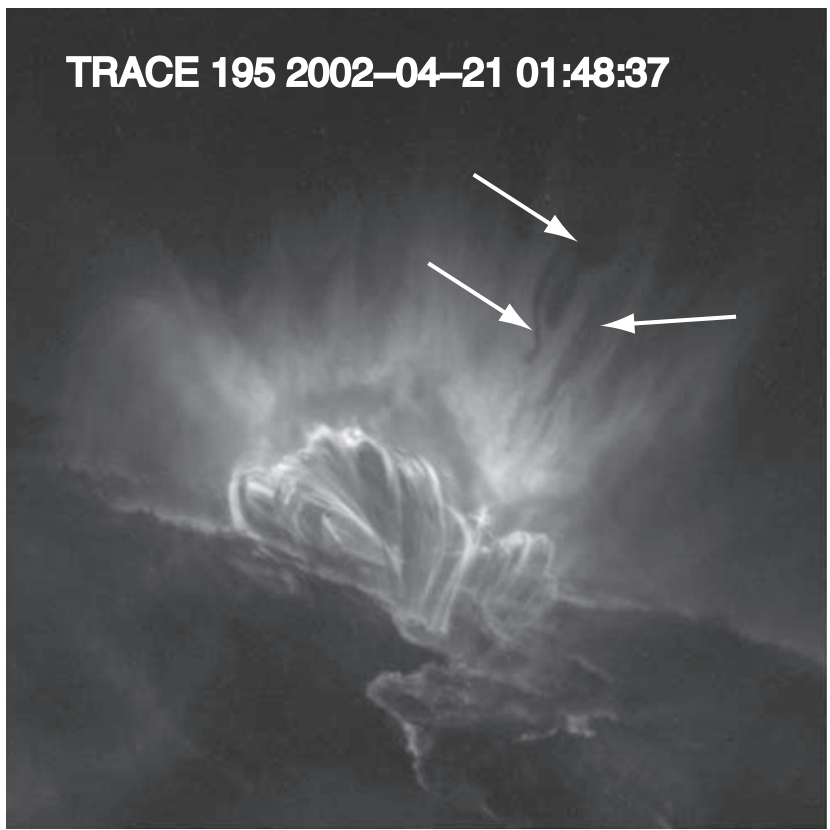

Figure 5. Supra-arcade downflows observed by the 195 channel on TRACE during the 21 April 2002 X-flare. Arrows mark some of the dark voids, which travel down toward the top of the arcade as the flare progresses. See Movie S1 of the flare in the auxiliary material.

stage process that begins with a thermal expansion, but nonthermal acceleration via waves generated by the unstable electron beams and via field aligned potential drops plays the more important role. The accelerated ions contribute significantly to the plasma population in the inner plasma sheet and middle magnetosphere, especially during disturbed times [see, e.g., Moore et al., 2007].

[15] A similar process occurs in solar flares. Flare loops in the corona are anchored in extremely dense, cool chromospheric plasma. During the flare, the chromospheric plasma gets heated, creating a pair of flare ribbons in the chromosphere made visible by this process. The heated chromospheric plasma expands to fill the flux tube, a process commonly referred to as chromospheric evaporation (but more accurately termed chromospheric ablation). The flare ribbons are in several respects analogous to the two auroral zones, one in each hemisphere on Earth [Akasofu, 1979]. In both cases the cooler upflowing plasma is an important component of the flux tube population, though in both the terrestrial and solar cases this filling process takes much longer than the field line shrinkage [see, e.g., Lin, 2004].

\section{Supra-arcade Downflows and Bursty Bulk Flows}

[16] Recent advances in imaging instruments that observe the solar corona have afforded the opportunity to observe new phenomena in solar flares. In particular, dark downflowing voids, commonly referred to as coronal voids or supra-arcade downflows, have been observed above postflare loops in the corona. These features are believed to be empty reconnected flux tubes collapsing through the current sheet after reconnection [McKenzie and Hudson, 1999; McKenzie, 2000]. Spectroscopic observations of these downflows have confirmed that they are voids, e.g., lowdensity regions, rather than absorption features [Innes et al., 2003a].

[17] An excellent example of these downflows was observed in the $195 \AA$ channel with the Transition Region and Coronal Explorer (TRACE) [Sheeley et al., 2004] and is shown in Figure 5. The TRACE 195 channel is mostly sensitive to plasma at around $1.5 \mathrm{MK}$, but the waveband also contains a high-temperature response that allows for imaging of the hot plasma above the flare loops. Dark voids are marked on the figure with arrows. These voids move downward towards the top of the flare arcade as the event progresses. This motion can be clearly seen in the movie of the event (Movie S1, included in the auxiliary material of this paper). ${ }^{1}$

[18] The magnetosphere is a very different plasma environment than the corona, and yet a similar phenomenon to supra-arcade downflows is observed after the onset of a substorm. High-speed, transient earthward flows, termed bursty bulk flows (BBFs) by Angelopoulos et al. [1992], have been inferred from observations by in situ spacecraft in the magnetosphere. These bursty bulk flows are sometimes associated with plasma-depleted flux tubes (or "bubbles" [see Sergeev et al., 1996]), making them surprisingly similar to the supra-arcade downflows observed during solar flares.

[19] The speeds of supra-arcade downflows and bursty bulk flows have been well measured. For the coronal flare shown in Figure 5, the observed downflows have initial speeds of 100-600 km s${ }^{-1}$ [Sheeley et al., 2004]. Other flares have associated downflows with similar measured speeds [McKenzie and Hudson, 1999; McKenzie, 2000]. It should be noted that the downflows are harder to detect the higher they are in the corona, so their initial speeds could be even higher than those reported here. Bursty bulk flow events in the magnetosphere are defined as having speeds of $>400 \mathrm{~km} \mathrm{~s}^{-1}$ [Baumjohann et al., 1990; Angelopoulos et al., 1994]. The "bubbles" observed by Sergeev et al. [1996] have speeds on the order of $100-400 \mathrm{~km} \mathrm{~s}^{-1}$.

[20] Spectroscopic measurements of the solar flare shown in Figure 5 have also revealed Doppler shifts indicative of high-speed plasma flows orthogonal to the supra-arcade downflows [Innes et al., 2003b]. These speeds were measured at $800-1000 \mathrm{~km} \mathrm{~s}^{-1}$, and the authors suggest that these velocities could be a result of reconnection in the wake of the downflow or shocks associated with the interaction between the plasmoid and the flare arcade, although the high magnitude of the speeds is not completely understood. Bursty bulk flows can also be associated with substantial plasma flows across the magnetotail in the dawn-dusk direction [Baumjohann et al., 1990; Nakamura et al., 2001]. These flows, which can be several hundred $\mathrm{km} \mathrm{s} \mathrm{s}^{-1}$, are interpreted as diversions of the earthward flowing plasma associated with compression in the magnetic field near the Earth [Nakamura et al., 2001], an explanation similar to the colliding plasmoid argument used by Innes et al. [2003b] to explain the strong orthogonal flows in the corona mentioned above.

${ }^{1}$ Auxiliary materials are available in the HTML. doi:10.1029/ 2008JA013049. 


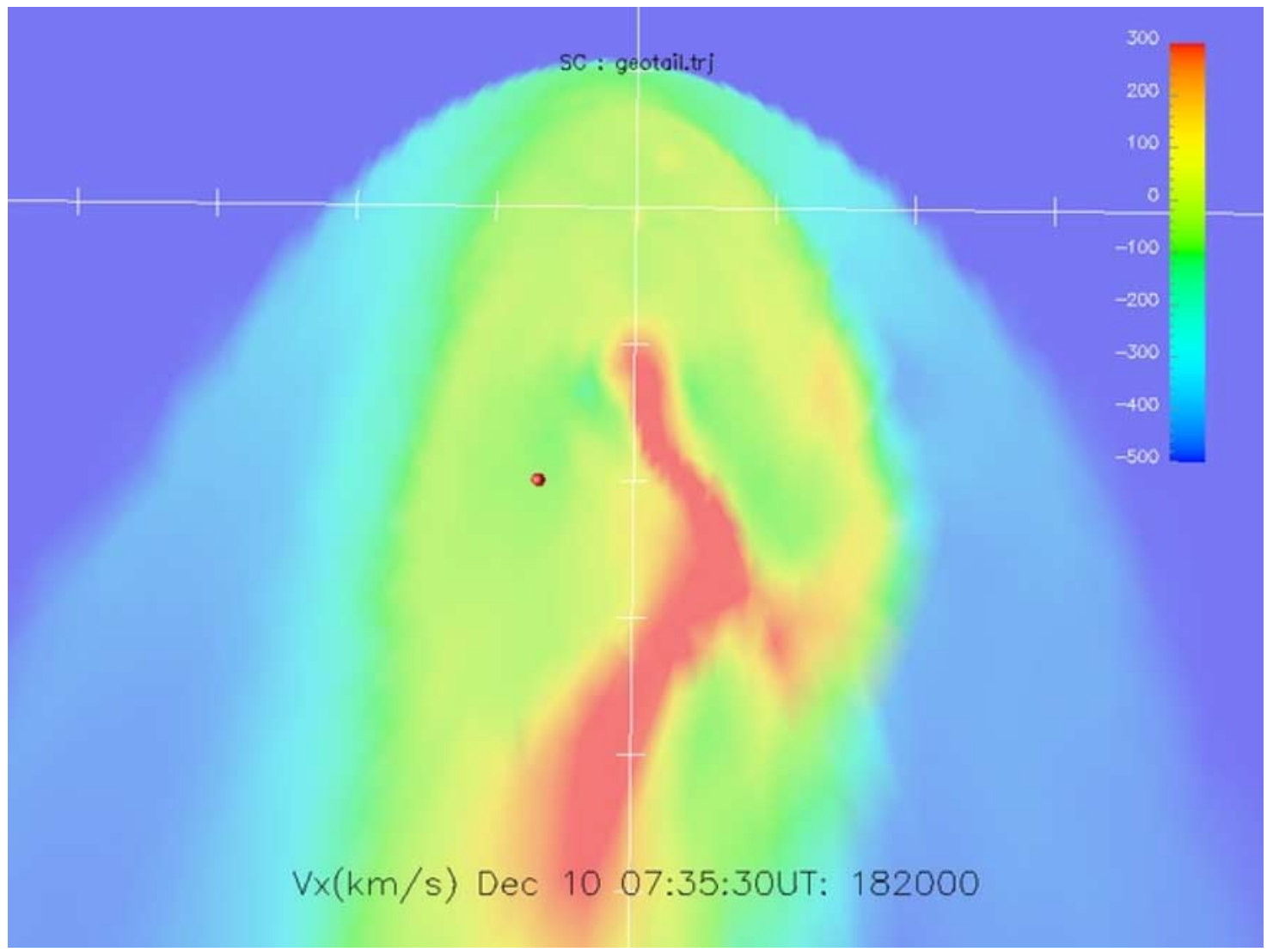

Figure 6. One representative flow channel (red) propagates from the tail into the inner magnetosphere of a global MHD simulation (from Guild [2006]). The figure shows the magnitude of the $\mathrm{X}_{G S M}$ plasma velocity from above the north pole, with the Sun to the top White GSM coordinate X, Y axes have tick marks every $10 \mathrm{R}_{E}$. The red dot marks the location of the Geotail satellite at the time of the modeled substorm. Please see Movie S2 of the entire substorm simulation in the auxiliary material.

[21] The supra-arcade downflows observed during the flare shown in Figure 5 rapidly decelerate as they descend toward the top of the flare arcade, as shown by Sheeley et al. [2004]. This deceleration is probably due to the braking of the shrinking loop caused by the loops underneath it. There is evidence for a similar phenomenon in the magnetosphere. Studies of bursty bulk flows have shown that the occurrence rate for events above $400 \mathrm{~km} \mathrm{~s}^{-1}$ decreases as the distance from the Earth becomes smaller [Baumjohann et al., 1990; Angelopoulos et al., 1994], which could be an indication that BBFs slow down as they get closer to the Earth. In addition, Shiokawa et al. [1997] found that high-speed flows were less common closer to the Earth and that this low occurrence rate is consistent with braking at the boundary between the dipolar and tail-like field structures due to an increased pressure gradient there.

[22] The cross-sectional areas of supra-arcade downflows and bursty bulk flows seem remarkably similar. The scale size of BBF events studied by Angelopoulos et al. [1997] using the WIND and GEOTAIL spacecraft was found to be less than $3 \mathrm{R}_{E}$. Sergeev et al. [1996] found that the cross-tail size of the bubbles was similar, on the order of $1-3 \mathrm{R}_{E}$. Using this range for the diameter of a circular cross section gives an area of $0.6-6 \times 10^{8} \mathrm{~km}^{2}$. A statistical analysis of the supra-arcade downflows in the corona for several events, including the one shown in Figure 5, places the areas of these events in a similar range $\left(0.2-1.2 \times 10^{8} \mathrm{~km}^{2}\right)$ (D. E. McKenzie and S. Savage, Quantitative examination of supra-arcade downflows in eruptive solar flares, submitted to Astrophysical Journal, 2008.)

[23] Because observations in the magnetotail are necessarily constrained to point observations at individual spacecraft, 3-D MHD simulations are extremely valuable for comparing the morphology of BBFs to that of supra-arcade downflows in the corona. Wiltberger et al. [2000] have successfully reproduced the characteristics of BBFs using a MHD simulations, including their elevated earthward velocities and accompanying dipolarizations. Figure 6 illustrates one example of a fast, localized earthward flow occurring in a recent substorm simulation. In Figure 6 the equatorial plane of the magnetosphere is colored with the magnitude of the $\mathrm{X}_{G S M}$ plasma velocity, highlighting the fast earthward flow (red) penetrating a largely stagnant plasma sheet and inner magnetosphere (green-yellow). White tick marks are shown every $10 \mathrm{R}_{E}$ for scale. Guild [2006] has demonstrated that these simulated fast flow channels have similar properties to observations of BBFs and represent the transport of underpopulated flux tubes into the inner magnetosphere. Comparing the morphology of the flow channels in these simulations with the image of the supra-arcade downflows in Figure 5 shows similarities in structure between the two phenomena. 
[24] Early models of bursty bulk flows in the magnetosphere concentrated on the effect of the interchange instability on a bubble of plasma with a lower entropy per unit magnetic flux, $P V \gamma$, than the surrounding plasma [Pontius and Wolf, 1990; Chen and Wolf, 1993]. Here, $P$ is the plasma pressure along the field line, $V$ is the volume per unit magnetic flux, and $\gamma$ is the polytropic index. Other authors have invoked intermittent or "patchy" reconnection, as in the model of Semenov et al. [1992], to explain bursty bulk flow events [Baumjohann et al., 1990; Sergeev et al., 1995; Shay et al., 2003].

[25] Chen and Wolf [1999], building on the earlier work of Pontius and Wolf [1990] and Chen and Wolf [1993], found that the velocity of the flux tubes perpendicular to the magnetic field is approximately the Alfvén speed and that there is a slow mode shock propagating parallel to the magnetic field. They also astutely point out that the bubble model and the patchy reconnection model are not mutually exclusive; bubbles could easily be formed by patchy reconnection events in the magnetotail. Patchy reconnection events are studied in the context of the solar corona by Linton and Longcope [2006], who use a thin flux tube model to simulate reconnection and the formation of downward moving flux tubes in the corona after a solar flare. They find that the velocity of the flux tube is a significant fraction $(45-82 \%)$ of the Alfvén speed for low- $\beta$ plasma conditions such as those in the corona.

[26] The studies discussed above show that the Alfvén speed is a determining factor in the behavior of systems involving reconnection. Looking at the plasma parameters given in Table 1, we see that the magnetosphere is an environment with low magnetic field strengths and low particle densities, while the densities and magnetic field strengths are orders of magnitude higher in the corona of the Sun. Nevertheless, the Alfvén speeds are similar in the two regimes, and thus the speeds of bursty bulk flows and supraarcade downflows are similar. This observation leads to the general conclusion that processes governed by the Alfvén speed should exhibit similar behaviors in these two (and possibly other) environments, while processes governed by other factors may be very different.

\section{Discussions and Conclusions}

[27] We have shown that the time and spatial scales of postreconnection phenomena in the solar corona and the magnetosphere are similar to within an order of magnitude. This result is surprising at first, owing to the very different plasma environments that exist in the magnetosphere and the corona. However, it turns out that the Alfvén speed is a governing parameter for the speeds of bursty bulk flows in the magnetosphere and the supra-arcade downflows in the corona, and it happens that the Alfvén speeds are similar in these two regimes.

[28] The similarities between the velocities, deceleration profile and size scales of bursty bulk flow events and supraarcade downflows strongly suggest that these phenomena are the same. This result is significant because it helps to rule out alternate interpretations in one regime or the other. For example, the supra-arcade downflows in the corona are observed as a void whose extent grows down toward the solar surface. It is possible that high velocities evacuate the region by ejecting plasma upward (radially away from the Sun), leaving a void whose lower tip is the region where the acceleration begins. This interpretation would be consistent with the velocity information derived from the spectroscopic data and with the image data for these events. However, since a similar phenomenon is observed in the magnetosphere, where plasma velocities can be measured and verified to be flowing earthward, we can be more confident that the interpretation of coronal plasma downflows is the correct one. In this case, identification of the solar events with the magnetospheric ones lends evidence to the idea that there is a downward flow of plasma and fields in the coronal case.

[29] Although the supra-arcade downflows observed in the corona show striking similarities to the density-depleted bubbles observed by Sergeev et al. [1996], some observations of BBFs in the magnetosphere show no density or pressure depletions at all [Angelopoulos et al., 1992]. Recent models of the "bubbles" in the magnetosphere have shown that depleted entropy per unit magnetic flux is a necessary feature in order to accelerate the bubbles toward the Earth [Birn et al., 2004]. This depletion can manifest in the form of a reduction in the flux tube volume, so a reduction in density is not a necessary condition for the fast earthward flows to take place. If similar conditions prevail on downflows in the corona, then it is possible that not all downflows are density depleted in the corona as well. For example, recent observations have found bright downflows in the corona after a filament eruption [Tripathi et al., 2007]. More study is needed to understand these observations in light of what we know about supra-arcade downflows in the corona and bursty bulk flows in the magnetosphere.

[30] Comparing these posteruption phenomena in the magnetosphere and the corona can be illuminating because the plasma regimes are so different, and conclusions that seem valid in one environment can be shown to be false when the same phenomenon is examined in another environment. For example, calculations by $M$. S. Nakamura et al. [2002] suggest that the size scale of bursty bulk flow events in the magnetosphere is related to the ion inertial length or the ion Larmor radius. They find that the minimum size for the bubbles is on the order of 10-20 times the ion inertial scale length, which works out to be about 3000 $6000 \mathrm{~km}\left(\sim 0.5-1 \mathrm{R}_{E}\right)$, a reasonable size scale for these events given the observations. In the corona, however, the ion inertial length and the ion Larmor radius are on the order of $1 \mathrm{~m}$, much smaller than the observed size scale of the supra-arcade downflows. Thus in the coronal case at least, the ion inertial length cannot be the governing factor in the size of the observed downflows. Some recent work [Lin et al., 2007; Riley et al., 2007] suggests that the size scale of the downflows in the corona is governed by the tearing mode instability in the current sheet. However, the tearing mode in the corona is resistive, while plasma in the geotail is collisionless. Further work is needed to see if the similarity in length scales is coincidental or governed by similar physics. It is also possible that higher spatial resolution coronal observations will reveal smaller-sized downflows.

[31] In this paper we have compared posteruption phenomena in the corona and the magnetosphere, two environ- 
ments with very different plasma properties. The motivation for comparing these phenomena is to establish whether the gross features of the plasma dynamics are universal. We have seen in this paper the emergence of such a universal process: the onset of reconnection associated with magnetic reconfiguration in both the corona and magnetosphere has similar consequences in both regimes. The posteruption plasma dynamics in the two cases show remarkable similarities and some marked differences that can be accounted for by the plasma parameters in each environment. Thus, we have shown that there are physical analogs between posteruptive phenomena in the magnetosphere and the corona associated with magnetic reconnection in both plasma environments.

[32] Acknowledgments. The authors would like to thank the scientists in the New England Space Science Consortium (NESSC) for helpful input and discussions, and the anonymous referees for insightful comments on the manuscript. K. Reeves and K. Korreck are supported by the NASA Solar-B Phase E contract to SAO, NNM07AA02C, and the TRACE contract to SAO from Lockheed Martin. J. Lin acknowledges support from NASA grant NNX07AL72G. This work was partially supported by CISM, an NSF Science and Technology Center funded under agreement ATM-0120950.

[33] Amitava Bhattacharjee thanks Boon-Chye Low and Spiro Antiochos for their assistance in evaluating this paper.

\section{References}

Akasofu, S.-I. (1979), Magnetospheric substorms and solar flares, Sol. Phys., 64, 333-348.

Akasofu, S.-I. (1985), Energy supply processes for magnetospheric substorms and solar flares - Tippy bucket model or pitcher model?, Astrophys. Space Sci., 108, 81-93.

Angelopoulos, V., W. Baumjohann, C. F. Kennel, F. V. Coronti, M. G. Kivelson, R. Pellat, R. J. Walker, H. Luehr, and G. Paschmann (1992), Bursty bulk flows in the inner central plasma sheet, J. Geophys. Res., 97 , 4027-4039.

Angelopoulos, V., et al. (1994), Statistical characteristics of bursty bulk flow events, J. Geophys. Res., 99, 21,257-21,280.

Angelopoulos, V., et al. (1997), Magnetotail flow bursts: association to global magnetospheric circulation, relationship to ionospheric activity and direct evidence for localization, Geophys. Res. Lett., 24, $2271-$ 2274, doi:10.1029/97GL02355.

Apatenkov, S. V., et al. (2007), Multi-spacecraft observation of plasma dipolarization/injection in the inner magnetosphere, Ann. Geophys., 25, $801-814$

Baumjohann, W., G. Paschmann, and H. Luehr (1990), Characteristics of high-speed ion flows in the plasma sheet, J. Geophys. Res., 95, $3801-$ 3809.

Birn, J., J. Raeder, Y. Wang, R. Wolf, and M. Hesse (2004), On the propagation of bubbles in the geomagnetic tail, Ann. Geophys., 22, 1773-1786.

Biskamp, D. (2000), Magnetic Reconnection in Plasmas, Cambridge Univ. Press, Cambridge, U. K.

Chen, C. X., and R. A. Wolf (1993), Interpretation of high-speed flows in the plasma sheet, J. Geophys. Res., 98, 21,409-21,419.

Chen, C. X., and R. A. Wolf (1999), Theory of thin-filament motion in Earth's magnetotail and its application to bursty bulk flows, J. Geophys. Res., 104, 14,613-14,626, doi:10.1029/1999JA900005.

Coroniti, F. V. (1985), Explosive tail reconnection - The growth and expansion phases of magnetospheric substorms, J. Geophys. Res., 90, $7427-7447$.

Forbes, T. G. (1996), Reconnection theory for flares (Invited), in Magnetic Reconnection in the Solar Atmosphere, Conf. Ser., vol. 111, edited by R. D. Bentley and J. T. Mariska, p. 259, Astron. Soc. of the Pac., San Fransisco, Calif

Forbes, T. G., and L. W. Acton (1996), Reconnection and field line shrinkage in solar flares, Astrophys. J., 459, 330-341.

Guild, T. B. (2006), Comparative studies of multi-scale convective transport through the Earth's plasma sheet, Ph.D. thesis, Boston Univ., Boston, Mass.

Hiei, E., and A. J. Hundhausen (1996), Development of a coronal helmet streamer of 24 January 1992, in Magnetodynamic Phenomena in the Solar Atmosphere - Prototypes of Stellar Magnetic Activity, IAU Colloq. vol. 153, edited by Y. Uchida, T. Kosugi, and H. S. Hudson, p. 125, Int. Astron Union, Paris.
Hughes, W. J. (1988), Multisatellite observations of field-aligned current systems, Adv. Space Res., 8, 321-331, doi:10.1016/02731177(88)90146-9.

Hughes, W. J., and H. J. Singer (1985), Mid-latitude Pi 2 pulsations, geosynchronous substorm onset signatures and auroral zone currents on March 22, 1979 - CDAW 6, J. Geophys. Res., 90, 1297-1304.

Innes, D. E., D. E. McKenzie, and T. Wang (2003a), SUMER spectral observations of post-flare supra-arcade inflows, Sol. Phys., 217, 247265 .

Innes, D. E., D. E. McKenzie, and T. Wang (2003b), Observations of 1000 km s-1 Doppler shifts in $107 \mathrm{~K}$ solar flare supra-arcade, Sol. Phys., 217, $267-279$.

Lin, J. (2004), Motions of flare ribbons and loops in various magnetic configurations, Sol. Phys., 222, 115-136, doi:10.1023/B:SOLA. 0000036875.14102 .39

Lin, J., J. Li, T. G. Forbes, Y.-K. Ko, J. C. Raymond, and A. Vourlidas (2007), Features and properties of coronal mass ejection/flare current sheets, Astrophys. J., 658, L123-L126, doi:10.1086/515568.

Linton, M. G., and D. W. Longcope (2006), A model for patchy reconnection in three dimensions, Astrophys. J., 642, 1177-1192, doi:10.1086 500965.

Liou, K., C.-I. Meng, A. T. Y. Lui, P. T. Newell, and S. Wing (2002), Magnetic dipolarization with substorm expansion onset, J. Geophys. Res., 107(A7), 1131, doi:10.1029/2001JA000179.

McKenzie, D. E. (2000), Supra-arcade downflows in long-duration solar flare events, Sol. Phys., 195, 381-399.

McKenzie, D. E., and H. S. Hudson (1999), X-Ray observations of motions and structure above a solar flare arcade, Astrophys. J., 519, L93-L96, doi: $10.1086 / 312110$.

McPherron, R. L., C. T. Russell, and M. P. Aubry (1973), Satellite studies of magnetospheric substorms on August 15, 1968: 9. Phenomenological model for substorms, J. Geophys. Res., 78, 31313149 .

Moore, T. E., M.-C. Fok, D. C. Delcourt, S. P. Slinker, and J. A. Fedder (2007), Global aspects of solar wind ionosphere interactions, J. Atmos. Terr. Phys., 69, 265-278, doi:10.1016/j.jastp.2006.08.009.

Nakamura, M. S., H. Matsumoto, and M. Fujimoto (2002), Interchange instability at the leading part of reconnection jets, Geophys. Res. Lett., 29(8), 1247, doi:10.1029/2001GL013780.

Nakamura, R., W. Baumjohann, R. Schödel, M. Brittnacher, V. A. Sergeev, M. Kubyshkina, T. Mukai, and K. Liou (2001), Earthward flow bursts, auroral streamers, and small expansions, J. Geophys. Res., 106, 10,79110,802, doi:10.1029/2000JA000306.

Nakamura, R., et al. (2002), Motion of the dipolarization front during a flow burst event observed by Cluster, Geophys. Res. Lett., 29(20), 1942, doi:10.1029/2002GL015763.

Ohtani, S.-I. (1998), Earthward expansion of tail current disruption: Dualsatellite study, J. Geophys. Res., 103, 6815-6826, doi:10.1029/ 98JA00013

Pontius, D. H., Jr., and R. A. Wolf (1990), Transient flux tubes in the terrestrial magnetosphere, Geophys. Res. Lett., 17, 49-52.

Priest, E. R., and T. G. Forbes (2000), Magnetic Reconnection: MHD Theory and Applications, Cambridge Univ. Press, New York.

Priest, E. R., and T. G. Forbes (2002), The magnetic nature of solar flares, Astron. Astrophys. Rev., 10, 313-377.

Reeves, K. K., D. B. Seaton, and T. G. Forbes (2008), Field line shrinkage in flares observed by the X-Ray Telescope on Hinode, Astrophys. J., 675 , $868-874$.

Riley, P., R. Lionello, Z. Mikiæ, J. Linker, E. Clark, J. Lin, and Y.-K. Ko (2007), "Bursty" reconnection following solar eruptions: MHD simulations and comparison with observations, Astrophys. J., 655, 591-597, doi:10.1086/509913.

Semenov, V. S., I. Kubyshkin, V. V. Levedeva, M. V. Sidneva, H. K. Biernat, M. F. Heyn, B. P. Besser, and R. P. Rijnbeek (1992), Timedependent localized reconnection of skewed magnetic fields, J. Geophys. Res., 97, 4251-4263.

Sergeev, V. A., V. Angelopoulos, D. G. Mitchell, and C. T. Russell (1995), In situ observations of magnetotail reconnection prior to the onset of a small substorm, J. Geophys. Res., 100, 19,121-19,134, doi:10.1029/ 95JA01471.

Sergeev, V. A., V. Angelopoulos, J. T. Gosling, C. A. Cattell, and C. T. Russell (1996), Detection of localized, plasma-depleted flux tubes or bubbles in the midtail plasma sheet, J. Geophys. Res., 101, 10,81710,826, doi:10.1029/96JA00460

Shay, M. A., J. F. Drake, M. Swisdak, W. Dorland, and B. N. Rogers (2003), Inherently three dimensional magnetic reconnection: A mechanism for bursty bulk flows?, Geophys. Res. Lett., 30(6), 1345, doi:10.1029 2002GL016267.

Sheeley, N. R., Jr, H. P. Warren, and Y.-M. Wang (2004), The origin of postflare loops, Astrophys. J., 616, 1224-1231, doi:10.1086/425126. 
Shiokawa, K., W. Baumjohann, and G. Haerendel (1997), Braking of highspeed flows in the near-Earth tail, Geophys. Res. Lett., 24, 1179-1182, doi:10.1029/97GL01062.

Sui, L., and G. D. Holman (2003), Evidence for the formation of a largescale current sheet in a solar flare, Astrophys. J., 596, L251-L254, doi:10.1086/379343.

Sui, L., G. D. Holman, and B. R. Dennis (2004), Evidence for magnetic reconnection in three homologous solar flares observed by RHESSI, Astrophys. J., 612, 546-556, doi:10.1086/422515.

Svestka, Z. F., J. M. Fontenla, M. E. Machado, S. F. Martin, and D. F. Neidig (1987), Multi-thermal observations of newly formed loops in a dynamic flare, Sol. Phys., 108, 237-250.

Tripathi, D., S. K. Solanki, H. E. Mason, and D. F. Webb (2007), A bright coronal downflow seen in multi-wavelength observations: evidence of a bifurcating flux-rope?, Astron. Astrophys., 472, 633-642, doi:10.1051/ 0004-6361:20077707.

Veronig, A. M., M. Karlický, B. Vršnak, M. Temmer, J. Magdalenić, B. R. Dennis, W. Otruba, and W. Pötzi (2006), X-ray sources and magnetic reconnection in the X3.9 flare of 2003 November 3, Astron. Astrophys. 446, 675-690, doi:10.1051/0004-6361:20053112.
Wiltberger, M., T. I. Pulkkinen, J. G. Lyon, and C. C. Goodrich (2000), MHD simulation of the magnetotail during the December 10, 1996, substorm, J. Geophys. Res., 105, 27649-27664, doi:10.1029/ 1999JA000251.

T. B. Guild, The Aerospace Corporation, 15049 Conference Center Drive, Suite 600, Chantilly, VA 20151-3824, USA. (timothy.guild@ aero.org)

W. J. Hughes, N. Schwadron, and H. Spence, Department of Astronomy, Boston University, Boston, MA 02215,USA. (hughes@bu.edu; nathanas@ bu.edu; spence@bu.edu)

K. E. Korreck, J. Lin, J. Raymond, and K. K. Reeves, HarvardSmithsonian Center for Astrophysics, Cambridge, MA 02138, USA (kkoreck@cfa.harvard.edu; jlin@cfa.harvard.edu; jraymond@cfa.harvard. edu; kreeves@cfa.harvard.edu)

S. Savage, Department of Physics, Montana State University, Bozeman, MT 59717-3840, USA. (savage@physics.montana.edu)

D. F. Webb, Institute for Scientific Research, Boston College, St. Clements Hall 402, 140 Commonwealth Drive, Chestnut Hill, MA 02467, USA. (david.webb@hanscom.af.mil)

M. Wiltberger, High Altitude Observatory, National Center for Atmospheric Research, Boulder, CO 80301, USA. (wiltbemj@ucar.edu) 